\title{
Estimation of Optimum Number of Poles for Random Signal by Yule-Walker Method
}

\author{
Ahmed Al Amin*, Md. Shoriful Islam, K.M. Abdul Al Woadud, Md. Jahirul Islam, \\ Md. ImrulKayes \\ Uttara University, House-4\&6, Road-15, Sector-6, Uttara Model Town, Uttara, Dhaka-1230, Bangladesh \\ ${ }^{*}$ Corresponding author, e-mail: shaon04@gmail.com
}

\begin{abstract}
The Yule-Walker method is an effective method to estimate the system response or spectrum for random signal. Hence most of the noise and spurious signals are random in nature, so it is very convenient to estimate their spectrum by Yule-Walker method successfully. The Yule-Walker method is an autoregressive process to estimate the poles and errors also based on the number of poles for Wide Sense Stationary (WSS) process as well. Moreover the value of zero will be correspondingly calculated based on the poles in case of all poles model. The main concern of this paper is to analyze the YuleWalker method and estimate the poles and zero along with the error based on the number of poles for a random signal. Moreover analyze the results to find out the optimum number of poles for least possible error.
\end{abstract}

Keywords: Auto Regression (AR), Random Signal, White Noise, Wide Sense Stationary (WSS) process), Yule-Walker (Y-W) Method

Copyright $(2017$ Universitas Ahmad Dahlan. All rights reserved.

\section{Introduction}

Power Spectral Density Estimation plays a vital role in signal processing and communication engineering. However to evaluate the noise charecterstics, to determine the power spectral density of the noise is an important issue. There are different kinds of estimations are available to estimate the spectrum of the signal, such as Padê approximation, Prony and Shank's methods. However all of these are for deterministic signal. Therefore for random signal, Yule-Walker method is a good choice for random signal which is WSS in nature as well; moreover $\mathrm{Y}$-W method is much more efficient for lower error and variance as well [1-2]. In present the communication technology is developing rapidly day by day to serve the purposes of our daily life. So to get the maximum performance of the communication system it is very obvious to identify and eliminate the effect of noise accurately [3]. Moreover to estimate the power spectrum density for any random or stochastic signal is also challenging as well. However Yule-Walker method is very much appropriate for random signals. So in 2016 random signal frequency identification has been performed efficiently based on $Y-W$ method [4].

The main challenge of $\mathrm{Y}-\mathrm{W}$ method is to determine the optimum number of poles for minimum error. Because the big amount of error may cause problem to estimate the PSD in case of random signal [5]. Moreover the large amount of poles will not provide moderate error but only increase the complexity [6]. Hence it is the main challenge to determine the optimum number of poles for random signal whereas the error should be least for $\mathrm{Y}-\mathrm{W}$ method in case of random signal.

The main objective of this paper is to determine the optimum number of poles for random signal by using Yule-Walker method. Moreover the error should be lower as well. Hence firstly some previous research work regarding relevant field will be analyzed in segment 2. Accordingly a random signal will be generated which is stochastic signal and WSS in nature based on the response of causal Linear Time Invariant (LTI) system for white noise. Moreover a Y-W method will be implemented to determine the values poles, zero and error. Subsequently different numbers of poles will be provide for same random signal to determine the values of pole, zero and error by Yule-Walker method which will be describe in methodology part. Finally based on Y-W method the values of error for different number of poles will be calculated. 
Moreover for different number of poles analyze the error verses number of poles graph to determine the optimum number of the poles for the generated random signal.

\section{Litarature Review}

Yule-Walker method is an effective and accurate estimation method for Stochastic or Random signal. However different research work has been done for this method for different scenarios. In 2011 a method has been developed to determine the power spectral density estimation based on Yule-Walker AR Method for Tamil Speech Signal [7]. However in 2012 Bayesian analysis has been performed for Spectral ARMA model. This analysis also illustrates that Bayesian approach provides much accurate estimation in case of ARMA $(2,2)$ and ARMA $(4,2)$ models compared to other approaches [8]. Besides the estimation for power spectrum in case of wide sense stationary random process using ARMA process has been done in 2014. YW method has been used to determine the peak of the spectrum based on the variance [9]. Moreover at that time, the estimation approach of spectrum by AR estimation and conventional estimation for turbine vibration signals in case of industrial environment has been compared successfully. Y-W method has been used to estimate the spectrum of the signals generated by the vibration of turbine [10]. In addition in 2014 ranging system has been studied for pseudorandom continuous wave signal [11]. Moreover in 2015 simulation analysis has been done for interface circuits for piezoelectric energy harvesting for random signal as well [12]. Afterwards in 2015 an analysis has been completed to determination of spectrum for Additive White Gaussian Noise (AWGN) by minimum set of Yule-Walker Equations in comparison with least mean square method for the same signal [13]. So estimate the optimum number of poles in case of random signal is a vital role by Yule-Walker Method. In methodology segment the whole process of this work will be describe explicitly

\section{Methodology}

Yule-Walker Method is an effective way to determine the number of poles and zero for AR process. It is very suitable to detect the optimum number of poles in case of random signal. Moreover the error should be least to determine the poles and zero. So at first a white noise signal has been generated which is random in nature. In this paper equation 1 represents the data sequence of the response from a causal linear time-invariant system for white noise. In this case the variance of the random signal remains very low $(0.05)$ and this is also a WSS process.

$$
H(z)=\frac{b}{1+a(1) z^{-1}+\ldots a(p) z^{-p}}
$$

Afterwards the Yule-Walker method has been developed based on equation 2, where $p$ is the number of poles. Moreover $a_{p}(I)$ determines the values of poles and $r_{x}(k)$ is the statistical autocorrelation of $\mathrm{x}$. However $\delta(k)$ represents an impulse response [14].

$$
r_{x}(k)+\sum_{l=1}^{p} a_{p}(l) r_{x}(k-l)=\sigma v^{2}|b(0)|^{2} \delta(k)
$$

So in case of signal processing the equation should be in form of matrices, hence the matrices form of the equation is showed in equation 3.In addition here $\sigma$ is the standard deviation and $v$ represents the variance of the random signal [14-16].

$$
\left[\begin{array}{cccc}
r_{x}(0) & r_{x}(-1) & \ldots & r_{x}(-p) \\
r_{x}(1) & r_{x}(0) & \ldots & r_{x}(-p+1) \\
\ldots & \ldots & \ldots & \ldots \\
r_{x}(p) & r_{x}(p-1) & \ldots & r_{x}(0)
\end{array}\right]\left[\begin{array}{c}
1 \\
a_{p}(1) \\
\ldots \\
a_{p}(p)
\end{array}\right]=\sigma^{2}|b(0)|^{2}\left[\begin{array}{c}
1 \\
0 \\
\ldots \\
0
\end{array}\right]
$$


To find the values of poles $a_{p}(k)$ and zero $b(0)$ should be calculated effectively. To determine the values of poles $a_{p}(k)$, the equation 4 can be used which is modified form of equation 2 in case of all poles model $[14,17]$.

$$
\begin{gathered}
{\left[\begin{array}{cccc}
r_{x}(0) & r_{x}^{*}(1) & \ldots & r_{x}^{*}(p) \\
r_{x}(1) & r_{x}(0) & \ldots & r_{x}^{*}(p-1) \\
\ldots & \ldots & \ldots & \ldots \\
r_{x}(p) & r_{x}(p-1) & \ldots & r_{x}(0)
\end{array}\right]} \\
\hdashline \mathrm{R}_{\mathrm{x}} \\
\mathrm{r}_{1}
\end{gathered}
$$

Moreover $\mathrm{r} 1$ is the autocorrelation matrix and $\mathrm{R}_{\mathrm{x}}$ determines the conjugate symmetry of r1. However to determine the value of zero $b(0)$, Equation (5) can be used as well based on the values of poles $\left(a_{p}(l)\right)$. Whereas $\varepsilon_{p}$ determines the error in Equation $(5)$ as well $[14,15]$.

$$
\varepsilon_{p}=r_{x}(k)+\sum_{l=1}^{p} a_{p}(l) r_{x}(k-l)=\sigma v^{2}|b(0)|^{2}
$$

Moreover for all poles model, the error can be calculated based on Equation (6) [14].

$$
\varepsilon_{p}=r_{x}(k)+\sum_{l=1}^{p} a_{p}(l) r_{x}(k-l)
$$

Subsequently Figure 1 illustrates the block diagram of the system. Where a random signal will be generated which is specifically a white noise with low variance. Moreover the number of poles will be also provided by the user. The main function then calls the $\mathrm{Y}-\mathrm{W}$ function to determine the values poles, zero and error as well for each number of poles based on Equation (4, 5 \& 6).

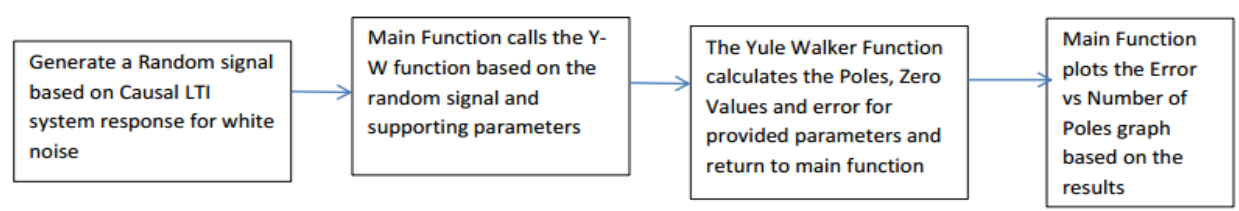

Figure 1. Block diagram of the System Principle

Afterwards the Y-W function returns the calculated parameters to the main function. Sequentially the main function illustrates the error verses number of poles graph to estimate the optimum number of poles of the provided random signal which is described in result analysis segment.

\section{Results and Analysis}

Based on the methodology in previous section a random signal has been generated which is WSS process and causal LTI response of white noise. Since the white noise is random 
in nature. Afterwards the numbers of poles are provided as input to the main function. Consequently the values of the poles and zeros along with the value of error are calculated by the functions which were developed based on the algorithm of theory. So at first a random signal of $9.5 \times 105$ samples is generated which is showed in Figure 2. However Figure 2 depicted the response of the causal LTI system for white noise which is wide sense stationary process.

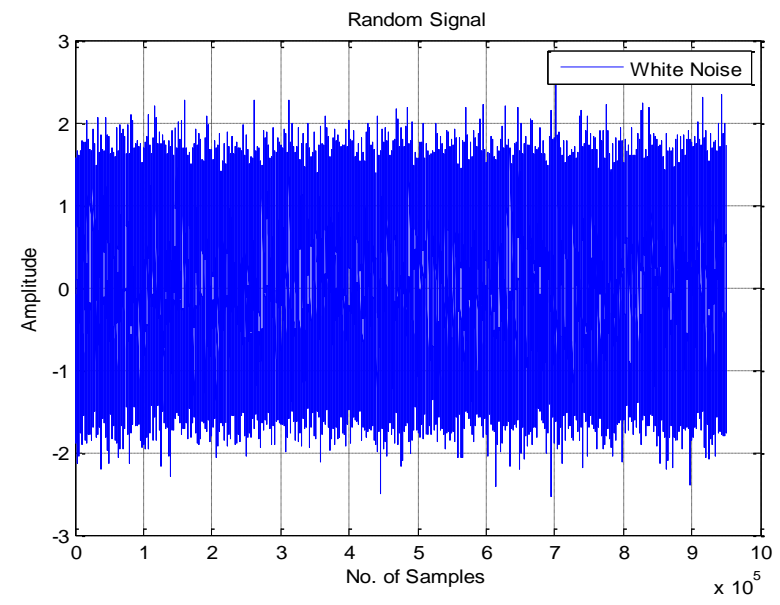

Figure 2. Generated Random Signal

By applying the Yule-Walker algorithm for two poles, the impact of error and number of poles are showed in figure 3 . The relation between number of poles and error is the novelty of this paper since this has not been shown for random signal in any previous research work.

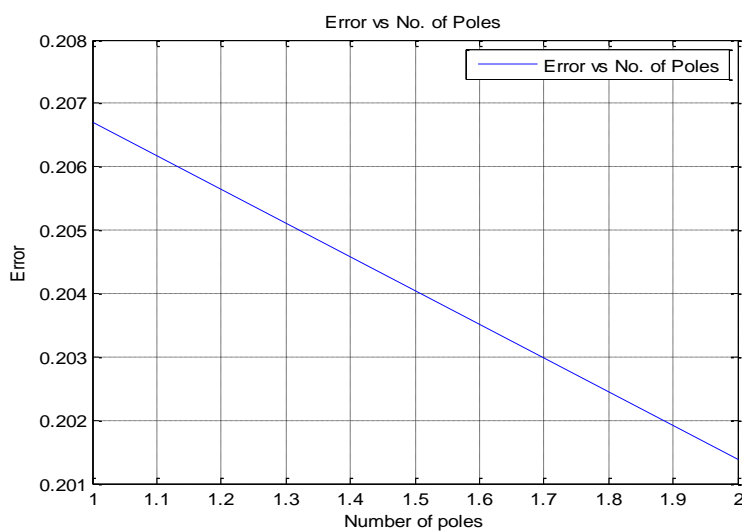

Figure 3. Impact of error with respect to the number of poles (No. of poles, $p=2$ )

Subsequently the numbers of poles are increasing to find out the optimum number of poles for least error. Figure 3 also illustrates that the error is very high for single number of pole, which is 0.2067 and for two poles the error decreases to almost 0.201. Now for higher number of poles such as four, the error decreases in significant way which is showed in Figure 4.

Afterwards Figure 4 demonstrates that for increasing number of poles such as two to three the error decreases significantly from 0.207 to 0.1667 . However for three to four poles the error decreases remarkably from 0.1667 to 0.159 , which is the minimum value of the error in case of four poles. Furthermore Figure 5 clarifies that for eight poles the error does not change anymore which remains constant from four to eight numbers of poles. 


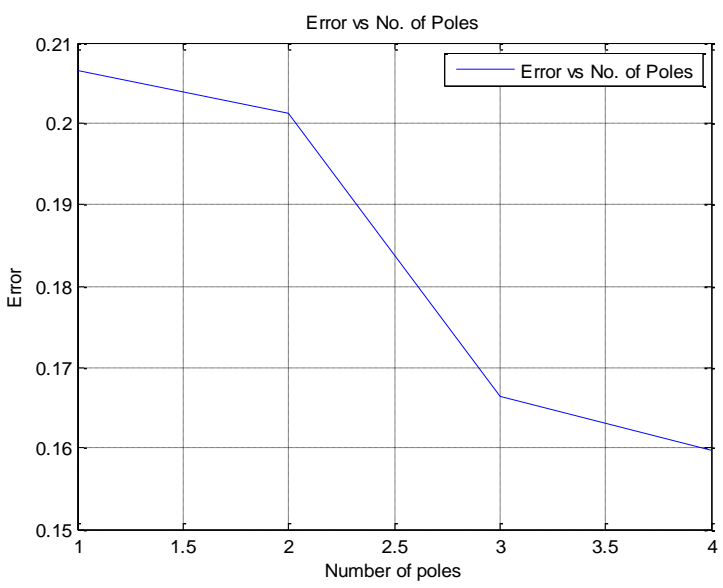

Figure 4. Impact of error with respect to the number of poles (No. of poles, $p=4$ )

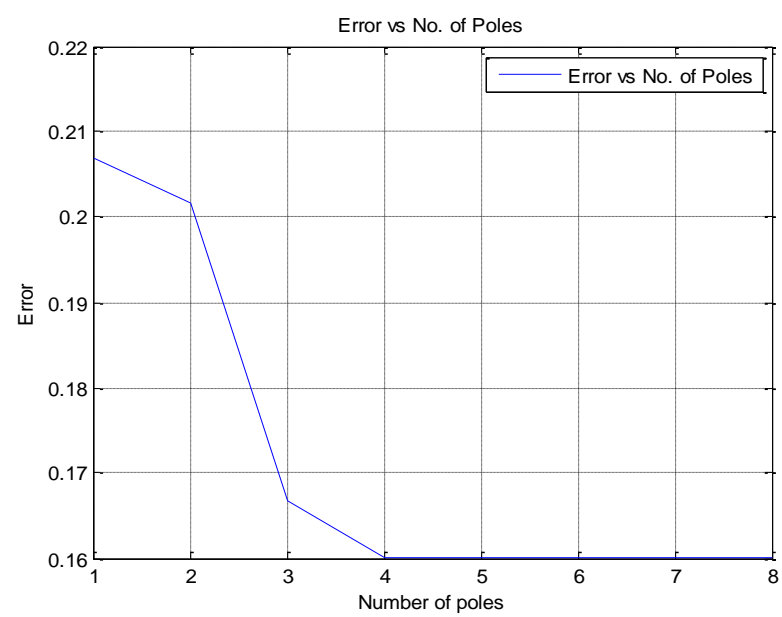

Figure 5. Impact of error with respect to the number of poles (No. of poles, $p=8$ )

Moreover for much higher number of poles such as sixteen poles the result of error remains same for the same random signal, which is illustrates in Figure 6 ornately. So this is clearly illustrates that the error does not varying after four number of poles for the same random signal.

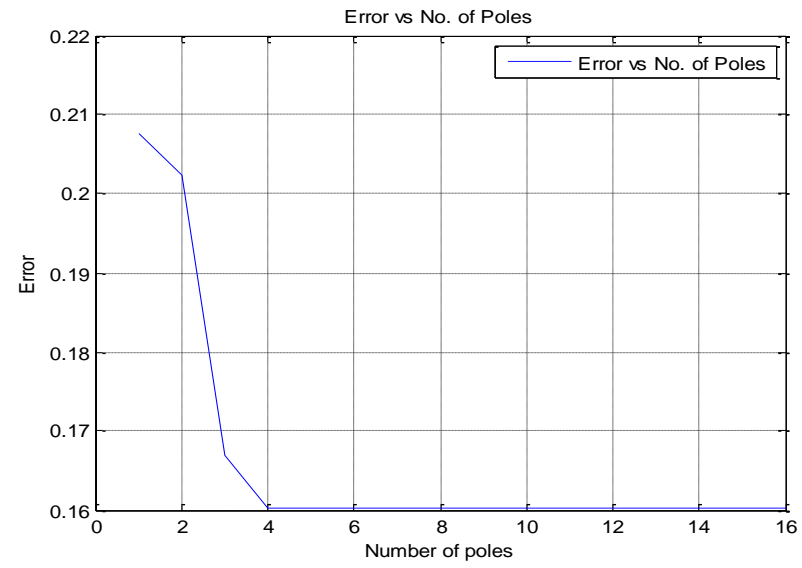

Figure 6. Impact of error with respect to the number of poles (No. of poles: $p=16$ ) 
Hence for four numbers of poles the value error is least and it does not change for higher number of poles accordingly. Hence in case of Yule-Walker method four is the optimum number of poles for this random signal. Moreover the values of poles, zero and minimum number of error are showed in Table 1 for 2, 4 and 8 number of poles since for higher poles the error remains same.

Table 1. Values of Poles, Zero for different number of poles

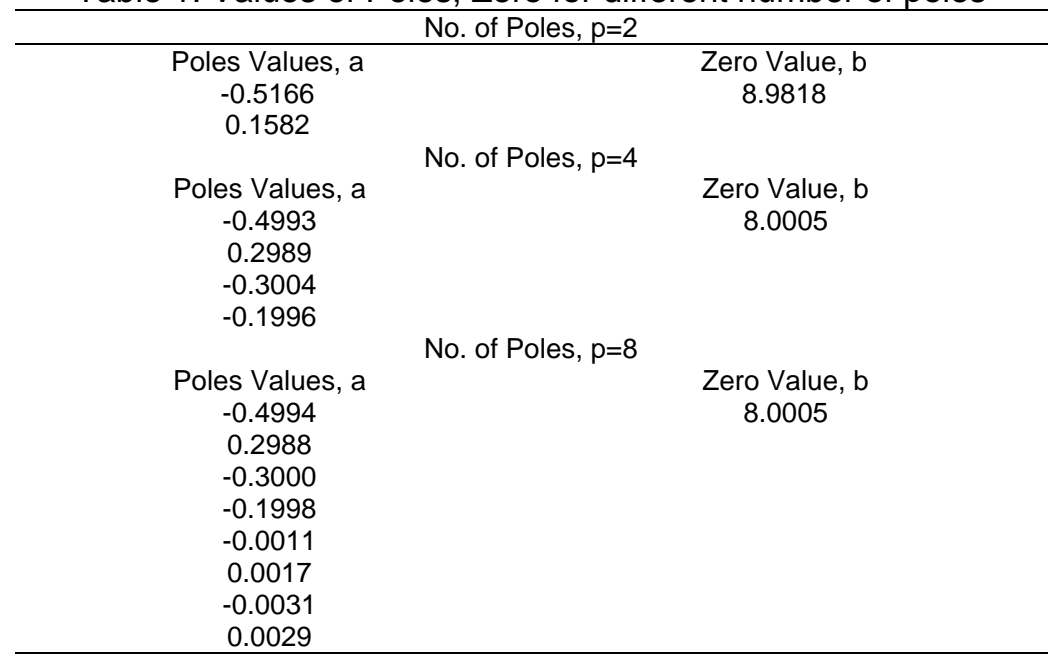

From the provided figures and the Table 1 it is clearly visualize that for Yule-Walker method in case of random signal, the error is minimum for four poles and beyond that. Hence sixteen or higher order of poles are not showed in Table 1. However the number of poles can be increase from one to any higher level. But the significant least value of error $(0.160)$ can be achieved for four poles which is the optimum number of poles for the specific white noise signal in case of $\mathrm{Y}-\mathrm{W}$ method. Nevertheless for higher number of poles such as eight, sixteen or higher the error remains unchanged which is showed in above analysis. In literature review this is clearly visible that previous works has been done based on Yule-Walker method, AR model and random signal for different application and methods. However this paper analyzed the relation between number of poles and error for random signal. Moreover to determine the optimum number of poles is another achievement of this paper for least error in case of random signal. Finally by using this optimum number of poles and zero values the spectrum estimation can be performed accurately due to least error.

\section{Conclusion}

Random signal is very complicated form of signal rather than deterministic signal. In addition white noise or random signal is Stochastic and Wide Sense Stationary Process in nature. So to derive the spectrum of random signal or to represent any system response based on random signal, Yule-Walker is the perfect choice for accurate estimation of optimum number of poles. Moreover the error can be also estimated for different number of poles for all poles model. So this paper estimates the optimum number of poles for a Yule-Walker method for a specific random signal. Hence the result analysis showed that among 2, 4, 8 and 16 numbers of poles for the same random signal, four is the optimum number of poles for the generated random signal based on the error verses number of poles graph. Besides the error is significantly low for four poles though it remains identical for higher amount of poles as well.

\section{References}

[1] CW Therrien. Discrete Random Signals and Statistical Signal Processing. 6th edition. New Jersey: Prentice-Hall. 1992: 123-127.

[2] B Friedlander, B Porat. The modified Yule-Walker methodof ARMA spectralestimation. Journal of IEEE Transactions on Aerospace and Electronic Systems. 1984; 20: 158-173. 
[3] Rob J Hyndman. Yule-Walker. Estimates ForContinous-Time Autoregressive Models. Journal of Time Series Analysis. 1993; 14: 281-296.

[4] Chunhuan Song, Random Signal Frequency Identification based on AR Model Spectral Estimation. International Journal on Smart Sensing and Intelligent Systems.2016; 9: 884-908.

[5] Pragati Sheel, et al. Parametric Method Based PSD Estimation using Gaussian Window. International Journal of Engineering Trends and Technology. 2015; 29: 19-22.

[6] Alimorad Mahmoudi. Adaptive Algorithm for Estimation of Two-Dimensional Autoregressive Fields from Noisy Observations. International Journal of Stochastic Analysis. 2014; 2: 1-5.

[7] V Radha, C Vimala, M Krishnaveni. Power Spectral Density Estimation Using Yule-Walker AR Method for Tamil Speech Signal. International Conference on Information Systems for Indian Languages, ICISIL. Springer Verlag. 2011; 2: 284-288.

[8] Silvestre Bezerra, Manoel I. Moala, Fernando Antonio lano, Yuzo. A Bayesian Analysis of Spectral ARMA Model. New York: Mathematical Problems In Engineering, Hindawi Publishing Corporation. 2012: 1-15.

[9] P Sasikiran, T Gowri Manohar, S Koteswara Rao. Estemating The Power Spectrum of a Wide Sense Stationary Random Process Using Parametric Approches (AR, MR). Journal of International Journal of Recent Advances in Engineering \& Technology. 2014; 2: 48-53.

[10] Junyou Huang. Study of Autoregressive (AR) Spectrum Estimation Algorithm for Vibration Signals of Industrial Steam Turbines. International Journal of Recent Advances in Engineering \& Technology. 2014; 7: 349-362.

[11] Jun Ma, Cheng-linBai. Study of Ranging System Using PseudoRandom Continuous Wave Signal. TELKOMNIKA Indonesian Journal of Electrical Engineering. 2014; 12: 1906-1910.

[12] Shuai Pang, Wenbin Li, Jiangming Kan. Simulation Analysis of Interface Circuits for Piezoelectric Energy Harvesting with Damped Sinusoidal Signals and Random Signals. TELKOMNIKA Indonesian Journal of Electrical Engineering. 2014; 13: 767-775.

[13] Abdelaziz Trabelsi, Otmane Ait Mohamed, Yves Audet. Robust Parametric Modeling of Speech in Additive White Gaussian Noise. Journal of Signal and Information Processing. 2015; 6: 99-108.

[14] Monson H Hayes. Statistical Digital Signal processing and Modeling. $3^{\text {rd }}$ edition. New Jersey: John Wiley and Sons. 1999: 160-165.

[15] MIS Bezerra. Precision of Yule-Walker methods for the ARMA spectral model. In Proceedings of the IASTED Conference on Circuits, Signals, and Systems. Clearwater Beach, Fla USA. 2002: 54-59.

[16] LV Dominguez. New Insights into the High-Order Yule-Walker Equations. IEEE Transactions on Acoustics, Speech and Signal Processing. 1990; 38: 1649-1651.

[17] RL Moses, V Simonyte, P Stoica, T Soderstrom. Anefficient linear method for ARMA spectral estimation. International Journal of Control. 1994; 59: 337-356. 